\title{
Socioeconomic and Racial Disparities in Diabetic Ketoacidosis Admissions in Youth With Type 1 Diabetes
}

\author{
Andrea R Maxwell, MD, MPH ${ }^{1 *}$, Nana-Hawa Yayah Jones, MD²,3, Stuart Taylor, MA4, Sarah D Corathers, MD², \\ Erika Rasnick, MS ${ }^{5}$, Cole Brokamp, PhD ${ }^{3,5}$, Carley L Riley, MD, MPP, MHS ${ }^{1,3}$, Allison Parsons, PhD ${ }^{1,3}$, \\ Jessica C Kichler, CDCES, PhD, CPsych ${ }^{6}$, Andrew F Beck, MD, MPH $3,4,7,8$
}

\begin{abstract}
${ }^{1}$ Division of Critical Care Medicine, Cincinnati Children's Hospital Medical Center, Cincinnati, Ohio; ${ }^{2}$ Division of Endocrinology, Cincinnati Children's Hospital Medical Center, Cincinnati, Ohio; ${ }^{3}$ Department of Pediatrics, University of Cincinnati College of Medicine, Cincinnati, Ohio; ${ }^{4}$ James M Anderson Center for Health Systems Excellence, Cincinnati Children's Hospital Medical Center, Cincinnati, Ohio; ${ }^{5}$ Division of Biostatistics and Epidemiology, Cincinnati Children's Hospital Medical Center, Cincinnati, Ohio; ${ }^{6}$ Department of Psychology, University of Windsor, Windsor, Ontario, Canada; ${ }^{7}$ Division of General and Community Pediatrics, Cincinnati Children's Hospital Medical Center, Cincinnati, Ohio; ${ }^{8}$ Division of Hospital Medicine, Cincinnati Children's Hospital Medical Center, Cincinnati, Ohio.
\end{abstract}

OBJECTIVE: We sought to determine whether census tract poverty, race, and insurance status were associated with the likelihood and severity of diabetic ketoacidosis (DKA) hospitalization among youth with type 1 diabetes (T1D).

METHODS: We conducted a retrospective populationbased cohort study using Cincinnati Children's Hospital electronic medical record (EMR) data from January 1 , 2011, to December 31, 2017, for T1D patients $\leq 18$ years old. The primary outcome was admission for DKA. Secondary outcomes included DKA severity, defined by initial $\mathrm{pH}$ and bicarbonate, and length of stay. Exposures were the poverty rate for the youth's home census tract, parent-reported race, and insurance status. We used multivariable logistic regression to analyze effects on odds of admission.

RESULTS: We identified 439 patients with T1D; 152 were hospitalized. The cohort was $48 \%$ female, $25 \%$ Black, and
$36 \%$ publicly insured; the median age was 14 years. For every $10 \%$ increase in a youth's census tract poverty rate, the adjusted odds of admission increased by $22 \%$ (95\% $\mathrm{Cl}, 1.03-1.47)$. Public insurance status was associated with DKA admission (adjusted odds ratio [AOR], 2.71, 95\% $\mathrm{Cl}, 1.62-4.55)$ while race was not. There were no clinically meaningful differences in $\mathrm{pH}$ or bicarbonate by census tract poverty, race, or insurance status; however, Black patients experienced differences in care (eg, longer length of stay).

CONCLUSION: Youth with T1D living in high poverty areas and on public insurance were significantly more likely to be admitted for DKA. Severity upon presentation was similar across exposures. Understanding contextual mechanisms by which disparities emerge will inform changes aimed at equitably improving care. Journal of Hospital Medicine 2021;16:517-523. () 2021 Society of Hospital Medicine
. ype 1 diabetes mellitus (T1D) is a common chronic condition of childhood. Its incidence has risen steadily over the past two decades. ${ }^{1}$ Treatment requires complex daily tasks, including blood glucose monitoring and insulin administration. The potential long-term complications of T1D_kidney failure, retinopathy, cardiovascular disease, and death-are grave but can be attenuated with effective glycemic management. ${ }^{2}$ Still, less than $25 \%$ of youths aged 13 to 19 years achieve target hemoglobin A1c (HbA1c) levels. ${ }^{3}$

Diabetic ketoacidosis (DKA) is an acute, life-threatening complication of T1D associated with suboptimal glycemic

*Corresponding Author: Andrea R Maxwell, MD, MPH; Email: amaxwell@ mcw.edu; Telephone: 414-805-6700; Twitter: @andrearmd.

Published online first August 18, 2021.

Find additional supporting information in the online version of this article.

Received: March 13, 2021; Revised: May 22, 2021; Accepted: June 6, 2021

(c) 2021 Society of Hospital Medicine DOI 10.12788/jhm.3664 management. In the United States, DKA hospitalizations increased during 2009-2014. ${ }^{4}$ One study found the average standardized cost of a DKA-related pediatric hospitalization exceeded $\$ 7000,{ }^{5}$ an amount not including missed school days, parent/caregiver workdays, and social and family disruptions. Costs extend to psychological well-being, with patients reporting that they "keep thinking [DKA] may happen again."6

The burden of T1D-related morbidity is not equitably distributed. Recent evidence suggests that racial/ethnic minorities and those with public insurance experience disproportionately high rates of DKA. ${ }^{7,8}$ Neighborhood socioeconomic measures, like poverty, add an important dimension illustrative of ecological conditions in which patients reside. These measures have been studied in relation to hospitalization rates for chronic conditions like asthma and heart failure. ${ }^{9,10}$ For example, one recent study found links between area-level poverty in the United States and the likelihood of readmissions for pediatric patients following an admission for DKA. ${ }^{11}$ We sought to build on this finding by investigating whether area-level poverty, which we measured as the census tract poverty rate, patient 
race, and insurance status were associated with the likelihood of initial DKA hospitalization and severity of DKA presentation.

\section{METHODS}

Design

We conducted a single-center, retrospective cohort study that examined data on youth with T1D extracted from the Cincinnati Children's Hospital Medical Center (CCHMC) electronic medical record (EMR). CCHMC is an urban, tertiary-care, freestanding pediatric hospital, with near-complete market penetration in Hamilton County, Ohio, particularly for subspecialty care. The racial and ethnic breakdown of Hamilton County's general population is 68\% White, 27\% Black/African American, $3 \%$ Asian, and 4\% Hispanic or Latino (adds to more than 100\% due to rounding). ${ }^{12}$ The CCHMC Institutional Review Board approved this study.

\section{Study Population}

We identified eligible patients through a clinical registry within the EMR, which includes all T1D patients seen at CCHMC within the preceding 24 months. Patients are added to this registry upon initial T1D diagnosis by a research nurse who evaluates autoantibody status and clinical presentation. All patients $\leq 18$ years old who had T1D, were active on the registry, and had an address within Hamilton County as of December 31, 2017, were included. Those with type 2 diabetes mellitus or secondary causes of diabetes were excluded. We captured patients on the registry as of December 31,2017, and then examined whether they had been hospitalized from January 1, 2011, to December 31, 2017.

\section{Covariates}

We extracted demographic and clinical data for patients within the T1D registry as of December 31, 2017, and, for those hospitalized, at the time of their admission. Specifically, we extracted date of birth (to calculate age at encounter), sex, and ethnicity. Age was treated as a continuous variable. We defined ethnicity as Latinx (inclusive of Hispanic) or non-Latinx. We calculated duration of T1D diagnosis for patients within the registry by taking the difference of the diabetes onset date and the end of the study period. We extracted whether registry patients had an insulin pump or a continuous glucose monitor (CGM). Hospitalized patients were identified as new-onset or established T1D by comparing their hospitalization with the T1D diagnosis onset date.

For the entire T1D registry, we also assessed a key clinical characteristic: a patient's median $\mathrm{HbA} 1 \mathrm{c}$. We enumerated the median $\mathrm{HbA} 1 \mathrm{c}$ of all patients' medians within the calendar year 2017, consistent with the methodology of a large international T1D registry. ${ }^{13}$ Among the subset of patients who were hospitalized, we obtained the $\mathrm{HbA} 1 \mathrm{c}$ value during or prior to hospitalization.

\section{Exposures}

We captured exposure variables from EMR documentation of patients' address, race, and insurance status. Given the small re- gional population of Latinx patients, we did not include ethnicity as an exposure. We used the address and insurance status at the beginning of the study period for established T1D patients, or at the time of diagnosis for new-onset patients, for analyses related to the likelihood of DKA admission. We used the address at the time of hospitalization for analyses of DKA presentation severity. We geocoded home addresses using ArcGIS software (Version 10.5.1; Esri), successfully geocoding $>95 \%$ to the street level and assigning patients to a corresponding census tracta census-defined geography consisting of approximately 4000 people. ${ }^{14}$ Census tracts, when drawn after the decennial (every 10 years) census, are designed to be sociodemographically homogenous $^{15}$ and have long been used in medical and public health research. ${ }^{16}$ We connected geocoded addresses to the census tract poverty rate, defined as the percentage of individuals within a tract living below the federal poverty level. For our analyses, we primarily treated census tract poverty as a continuous variable ranging from $0 \%$ to $100 \%$. We visualized the distribution of census tract poverty across Hamilton County using a choropleth map (Figure, part A). We also grouped the registry population into quartiles, ordered by census tract poverty levels, to ease the graphical display for Figure, part B. We defined race as White, Black (inclusive of African American), or other. We categorized insurance as Medicaid/public or Non-Medicaid/private.

\section{Outcomes}

The primary outcome was a hospitalization for DKA during the study period. We identified eligible events by admission diagnosis of DKA (International Classification of Disease, 10th Revision, Clinical Modification, E13.10 or E10.10). Secondarily, we assessed admission severity for each patient's first DKA hospitalization using initial $\mathrm{pH}$ from a venous blood gas and initial bicarbonate from the basic metabolic panel. These measures were chosen in accordance with the American Diabetes Association's published classification of DKA severity, which uses $\mathrm{pH}$, bicarbonate, and mental status. ${ }^{17}$ We defined the time for correction as the minutes from admission time until the anion gap was $\leq 12$ or the bicarbonate was $\geq 18$. We calculated total inpatient bed-days and pediatric intensive care unit (PICU) bed-days, and identified whether a patient was readmitted for DKA during the study period.

\section{Statistical Analyses}

We used descriptive statistics to characterize demographic and clinical information for patients within the T1D registry and for the hospitalized subset. We examined DKA severity data for all admitted patients by diagnosis status (new-onset T1D vs established). Associations between categorical outcomes were assessed using the Chi-square or Fisher exact test, and continuous outcomes were evaluated using the Mann-Whitney Rank Sum test.

We individually examined the effects of census tract poverty, race, and insurance on the odds of hospitalization through logistic regression. After confirming overlap and variability in census tract poverty rates by race and admission status (Appendix Table 1), we included these exposures in a multivariable model to 

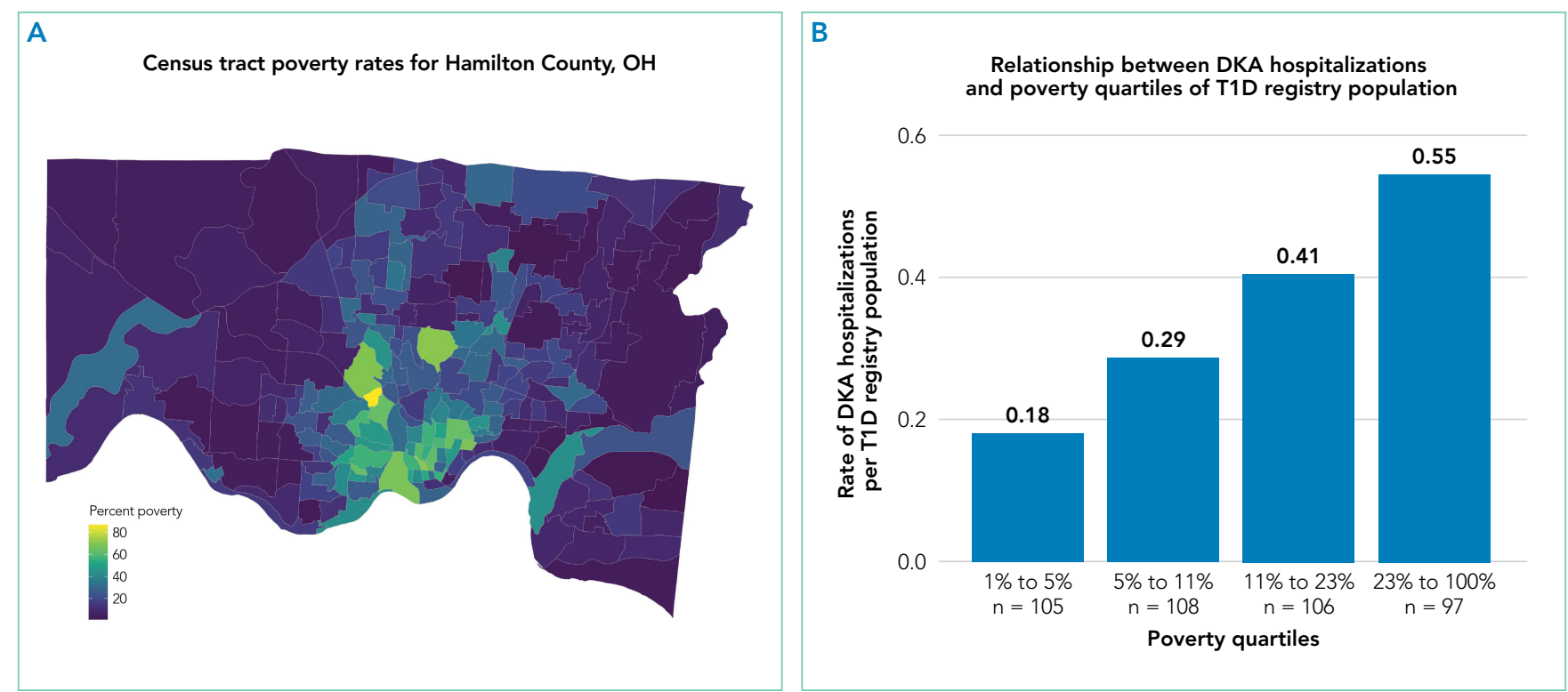

FIG. Distribution of Census Tract Poverty. (A) Choropleth Map of Hamilton County, Ohio. The map illustrates percent poverty displayed as a continuous variable based on census tract data. (B) The bar chart shows the number of diabetic ketoacidosis (DKA) hospitalizations per type 1 diabetes (T1D) registry population by poverty quartile.

capture their independent effects. Because the impact of census tract poverty, race, and insurance on hospitalization odds could vary depending on T1D diagnosis status, we conducted a sensitivity analysis by repeating the multivariable model with only established T1D patients.

Given that median $\mathrm{HbA} 1 \mathrm{c}$ levels differed for T1D registry patients based on census tract poverty, race, and insurance, we used linear regression in a post-hoc analysis to evaluate associations between those exposures and median $\mathrm{HbA} 1 \mathrm{c}$. Age, gender, ethnicity, and duration of diabetes were not included in the models given that these variables did not differ by outcome variables. Diabetes technology-CGM and insulin pumps-was not included as a confounder because we hypothesized it mediated the effect of area-level poverty. For analyses, SigmaPlot (Version 14.0; SPSS Inc.) and R statistical software were used (The R Project for Statistical Computing).

\section{RESULTS}

A total of 439 patients were in the T1D registry and lived in Hamilton County as of December 31, 2017. Their demographic characteristics are listed in Table 1. Their median age was 14 years; $48 \%$ were female. The median poverty rate of the census tracts in which these youth lived was $11 \%$. A total of $24.6 \%$ of patients identified as Black, $73.1 \%$ as White, and $2.3 \%$ as Hispanic; $35.8 \%$ were publicly insured. Patients had a median duration of T1D of 61 months (5 years, 1 month), with a median $\mathrm{HbA1c}$ (of all patients' medians) of $8.7 \%$. A total of $58.1 \%$ had a CGM, and $56 \%$ used an insulin pump.

\section{Hospitalization Characteristics of Those in the T1D Registry}

Approximately one-third of registry patients $(n=152)$ experienced $\geq 1$ hospitalization for DKA during the study period (inclusive of new-onset and established diagnosis). Age, gender, ethnicity, and duration of T1D diagnosis were similar between those who experienced a hospitalization and those who did not. The median census tract poverty rate was higher among those who were hospitalized compared to those who were not $(15 \%$ vs $8 \%, P<.01)$. Among hospitalized patients, $41 \%$ identified as Black; among all who were not hospitalized, $16 \%$ identified as Black $(P<.01)$. Among all hospitalized patients, $56.6 \%$ were covered by public insurance, while $24.7 \%$ of non-hospitalized patients were covered by public insurance $(P<.01)$. Hospitalized patients were more likely to have a higher median $\mathrm{HbA} 1 \mathrm{c}(9.5 \%$ vs $8.4 \%, P<.01)$ and were significantly less likely to have a CGM or insulin pump (both $P<.01$ ). These associations were magnified among the roughly $10 \%$ of the T1D registry population ( $n=42$ ) who experienced $\geq 2$ hospitalizations.

Figure, part $A$, is a choropleth map depicting the distribution of poverty by census tract across Hamilton County. The map is complemented by part B of the Figure, which displays the T1D registry population split into poverty quartiles and each quartile's DKA admission rate per T1D registry population. As census tract poverty increases, so too does the rate of DKA hospitalizations. There is an almost threefold difference between the hospitalization rate of the highest poverty quartile and that of the lowest.

\section{Likelihood of Hospitalization}

We examined the exposures of interest-census tract poverty, race, and insurance-and odds of hospitalization at a patient level using logistic regression models (Table 2). In the multivariable model that included these exposures, we found that for every $10 \%$ increase in the census tract poverty rate, the odds of being hospitalized for DKA increased by $22 \%$ (95\% Cl, 1.03-1.47). Race was not significantly associated with odds of DKA hospitalization in the multivariable model. Public insurance was associated with a 2.71-times higher odds of 
TABLE 1. Demographic and Clinical Characteristics of T1D Registry Patients and Comparison of Hospitalized Youth With Those Who Were Not Hospitalized

\begin{tabular}{|c|c|c|c|c|c|c|}
\hline Characteristic & $\begin{array}{l}\text { All registry patients, } \\
\text { median (IQR) } \\
\text { or No. }(\%) \\
(\mathrm{N}=439)\end{array}$ & $\begin{array}{l}\text { Not hospitalized, } \\
\text { median (IQR) } \\
\text { or No. (\%) } \\
(n=287)\end{array}$ & $\begin{array}{c}\text { Hospitalized } \\
\text { ( } \geq 1 \text { admission), } \\
\text { median (IQR) } \\
\text { or No. (\%) } \\
(n=152)\end{array}$ & $P$ value ${ }^{a}$ & $\begin{array}{c}\text { Rehospitalized } \\
\text { ( } \geq 2 \text { admissions), } \\
\text { median (IQR) } \\
\text { or No. (\%) } \\
(n=42)\end{array}$ & \\
\hline Age, y & $14(11-16)$ & $14(11-16)$ & $14(11-16)$ & 1 & $14.5(12.0-16.3)$ & .17 \\
\hline Female sex & $211(48.1)$ & $131(45.6)$ & $80(53)$ & .16 & $23(55)$ & .27 \\
\hline Census tract poverty & $11(5-23)$ & $8(4-19)$ & $15(7-31)$ & $<.01$ & $26(15-34)$ & $<.01$ \\
\hline Race & & & & $<.01$ & & $<.01$ \\
\hline Black & $108(24.6)$ & $46(16)$ & $62(41)$ & & $22(52)$ & \\
\hline White & $321(73.1)$ & $235(81.9)$ & $86(57)$ & & $18(43)$ & \\
\hline Other & $10(2.3)$ & $6(2.1)$ & $4(3)$ & & $2(5)$ & \\
\hline Ethnicity-Latinx & $10(2.3)$ & $5(1.7)$ & $5(3)$ & .32 & $3(7)$ & .07 \\
\hline Insurance & & & & $<.01$ & & $<.01$ \\
\hline Public & $157(35.8)$ & $71(24.7)$ & $86(56.6)$ & & 30 (71.4) & \\
\hline Private & $282(64.2)$ & $216(75.3)$ & $66(43.4)$ & & $12(28.6)$ & \\
\hline Duration of diabetes, $\mathrm{mo}^{\mathrm{c}}$ & $61(34-104)$ & $57(30-106)$ & $63.5(40.0-99.8)$ & .7 & $78(55.8-122.2)$ & $<.01$ \\
\hline Median HbA1c, \% & $8.7(7.8-10.1)$ & $8.4(7.7-9.4)$ & $9.5(8.4-11.4)$ & $<.01$ & $11.2(9.3-13.8)$ & $<.01$ \\
\hline \multicolumn{7}{|l|}{ Diabetes technology } \\
\hline Continuous glucose monitor & $255(58.1)$ & $186(64.8)$ & $69(45)$ & $<.01$ & $14(33)$ & $<.01$ \\
\hline Insulin pump & $246(56.0)$ & $186(64.8)$ & $60(39)$ & $<.01$ & $11(26)$ & $<.01$ \\
\hline $\begin{array}{l}\text { a Compares those who were not hospit } \\
\text { bCompares those who were not hospi } \\
\text { 'Duration from time of diagnosis to en } \\
\text { dMedian of each patient's median } \mathrm{Hb} / \\
\text { Abbreviation: IQR, interquartile ratio. }\end{array}$ & $\begin{array}{l}\text { ose who were hospitalize } \\
\text { ose who were rehospitaliz } \\
\text { riod } \\
\text { ast year }\end{array}$ & $\begin{array}{l}\text { admission) } \\
\geq 2 \text { admissions) }\end{array}$ & & & & \\
\hline
\end{tabular}

TABLE 2. Logistic Regression Models Assessing Association of Census Tract Poverty, Race, and Insurance With Odds of Hospitalization Among T1D Registry Youth

\begin{tabular}{|c|c|c|c|}
\hline Exposure & Odds ratio $(95 \% \mathrm{Cl})$ & Adjusted odds ratio $(95 \% \mathrm{Cl})^{\mathrm{a}}$ & $\begin{array}{l}\text { Adjusted odds ratio }(95 \% \mathrm{Cl}) \text { for youth } \\
\text { with established T1D diagnosis }\end{array}$ \\
\hline Census tract poverty rate $^{b}$ & $1.47(1.28-1.71)$ & $1.22(1.03-1.47)$ & $1.29(1.03-1.61)$ \\
\hline \multicolumn{4}{|l|}{ Race } \\
\hline Black & $3.39(2.14-5.41)$ & $1.32(0.71-2.43)$ & $0.88(0.37-2.01)$ \\
\hline White & $\operatorname{Ref}^{c}$ & $\operatorname{Ref}^{c}$ & $\operatorname{Ref}^{c}$ \\
\hline Other & $2.63(0.61-11.36)$ & $1.69(0.36-7.92)$ & $2.22(0.27-13.10)$ \\
\hline \multicolumn{4}{|l|}{ Insurance } \\
\hline Public & $4.07(2.65-6.29)$ & $2.71(1.62-4.55)$ & $3.24(1.62-6.46)$ \\
\hline Private & $\operatorname{Ref}^{c}$ & $\operatorname{Ref}^{c}$ & $\operatorname{Ref}^{c}$ \\
\hline
\end{tabular}


TABLE 3. Linear Regression Models Assessing Association of Census Tract Poverty, Race, and Insurance With Median $\mathrm{HbA1c}$ Among T1D Registry Youth

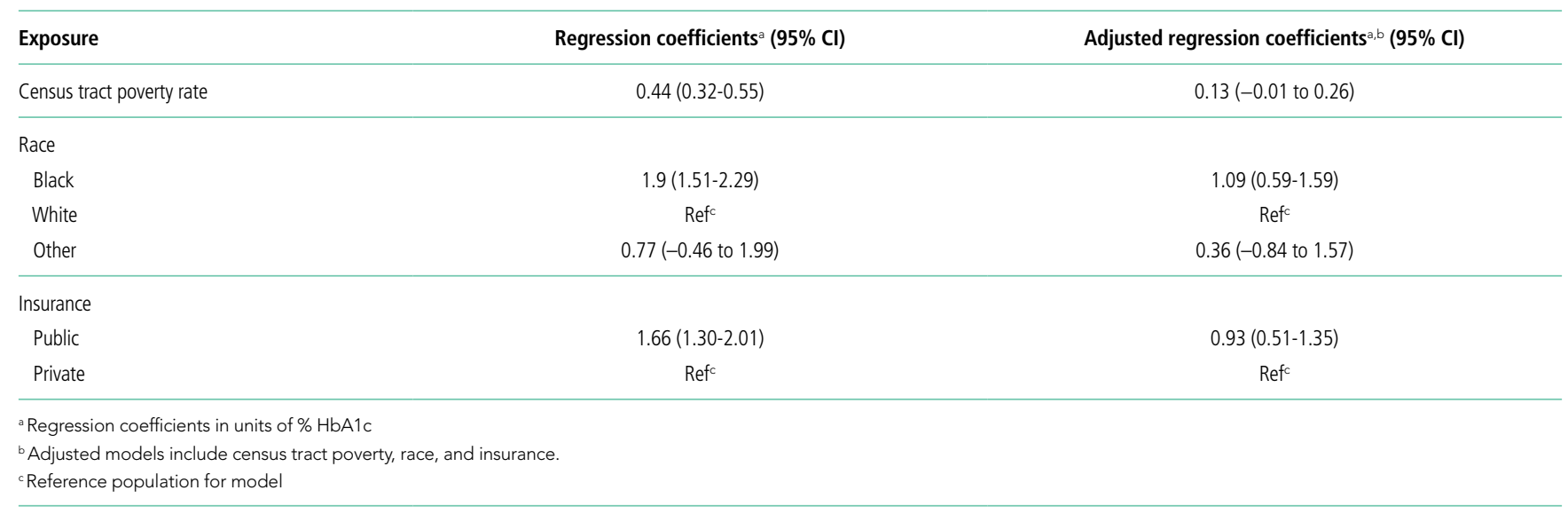

hospitalization compared to those with private insurance $(95 \%$ $\mathrm{Cl}, 1.62-4.55)$. Our sensitivity analysis of only those with established T1D diagnoses showed similar results to that of the entire T1D registry population.

\section{Severity of DKA Presentation}

A total of 152 registry patients were admitted; 89 patients had new-onset T1D, and 63 had an established T1D diagnosis. We examined presenting factors (initial $\mathrm{pH}$, initial bicarbonate, $\mathrm{HbA} 1 \mathrm{c}$ ) and clinical characteristics (time to correction, inpatient bed-days, PICU bed-days) by census tract poverty, race, and insurance for all hospitalized patients. There were no significant differences in presenting factors or outcomes by census tract poverty or insurance. However, we did note certain differences by race. Indeed, though initial $\mathrm{pH}$, bicarbonate, and time to correction did not meaningfully differ by race, Black patients had a longer length of stay than their White peers (Appendix Table $2 ; 3.06$ vs 2.16 days, $P<.01$ ). We further examined this by diagnosis status, as newly diagnosed patients often stay longer for diabetes education. ${ }^{18}$ Black patients had a longer length of stay than White patients, regardless of whether they had new-onset (3.85 vs 2.99 days, $P<.01$ ) or established T1D (1.83 vs 0.97 days, $P<.01$ ). Additionally, we found that the $\mathrm{HbA} 1 \mathrm{c}$ was significantly higher in Black versus White patients (12.4\% vs $10.8 \%, P=.01)$. HbA1c was significantly different between established T1D patients identifying as Black and those identifying as White $(11.7 \%$ vs $9.5 \%, P<.01)$; however, $\mathrm{HbA} 1 \mathrm{c}$ did not differ by race for newly diagnosed patients $(12.5 \%$ vs $13.0 \%, P=.63)$.

\section{Median HbA1c of All T1D Registry Patients}

Given the suggestion of $\mathrm{HbA} 1 \mathrm{c}$ variability by exposure variables, we performed a post-hoc linear regression analysis. Table 3 displays the linear regression models assessing associations of census tract poverty, race, and insurance on median $\mathrm{HbA} 1 \mathrm{c}$ for all T1D registry patients. In the multivariable model, census tract poverty was no longer associated with higher $\mathrm{HbA} 1 \mathrm{c}$ levels $(0.13,95 \% \mathrm{Cl},-0.01$ to 0.26$)$. However, Black patients continued to have significantly higher $\mathrm{HbA} 1 \mathrm{c}$ levels than White patients (1.09\% higher; $95 \% \mathrm{Cl}$, 0.59-1.59). Also, those with public insurance had significantly higher $\mathrm{HbA1c}$ levels than those with private insurance $(0.93 \%$ higher; $95 \% \mathrm{Cl}, 0.51-1.35)$.

\section{DISCUSSION}

Living in high poverty areas, identifying as Black, and having public insurance were each associated with a higher likelihood of hospitalization for DKA in an unadjusted model. When we examined these exposures together in a multivariable model, census tract poverty and insurance remained associated with a higher likelihood of hospitalization for DKA. Race-with a wide confidence interval—was no longer associated with a higher likelihood of hospitalization. The increased likelihood of hospitalization initially seen for Black patients may be because they are more likely to reside in high poverty areas and be on public insurance-inequities associated with structural racism.

These results mirror those of other studies that have found area-level poverty to be associated with higher rates of intensive care admissions and hospitalizations for conditions like asthma. ${ }^{10,19}$ Our findings are also similar to those of a previous study that found that area-level deprivation was associated with a greater likelihood of DKA readmissions in pediatric and adult patients, as well as an adult study that showed increased neighborhood deprivation was associated with higher odds of DKA hospitalization. ${ }^{11,20}$ Further examination of the mechanisms underlying DKA hospitalization is clearly warranted and would be informed by a deeper awareness of the social determinants of health-such as community context and the neighborhood/built environment ${ }^{21}$ —and their links to racial inequities.

Neighborhood socioeconomic status, here measured as census tract poverty, is likely linked to hospitalization for DKA through contextual factors like food insecurity, limited access to supportive care (eg, presence of a school nurse who can co-manage T1D with the patient/family and medical team), and adverse environmental exposures. ${ }^{22-24}$ To improve the health of patients, prevent hospitalizations, and achieve health 
equity, we suggest that both individual factors and these contextual factors need to be evaluated and addressed through community-connected interventions. ${ }^{25,26}$ For example, interventions that deploy community health workers to navigate the complexities of health systems have been effective in reducing $\mathrm{HbA} 1 \mathrm{c}$ levels in adults with type 2 diabetes mellitus ${ }^{27}$ and are beginning to be studied in pediatrics. ${ }^{28}$

Black patients had a higher median HbA1c compared to their White counterparts in the T1D registry. One study has previously shown that hemoglobin glycation biologically differed by around $0.5 \%$ in Black patients compared to White patients. ${ }^{29}$ Despite this purported biological difference, a greater difference in $\mathrm{HbA} 1 \mathrm{c}$ - nearly 2 points - was seen in the univariate model, illustrating that contextual factors such as socioeconomic status and treatment patterns, like the use of diabetes technology, exceed this biological difference. ${ }^{30-32}$ Our multivariable results support the importance of these contextual factors, as the $\mathrm{HbA} 1 \mathrm{c}$ difference decreased from 1.9 to 1.09 after census tract poverty and insurance were included.

Such differences by race also emerge from experiences of discrimination and bias. Thus, to improve outcomes and equity, we must understand and address racism and its many ill effects. Different levels of racism, such as structural or personally mediated racism, interact with social determinants of health in various ways. ${ }^{33,34}$ For example, redlining-racially discriminatory grading of neighborhoods' creditworthiness in the 1930s - is an example of structural racism that continues to affect the health of patients today. ${ }^{35,36}$ Black families are more likely to live in areas with higher poverty, even after controlling for household income level. ${ }^{37}$ The interaction between race and poverty is complex given these historical underpinnings of structural racism, as evidenced by race no longer being associated with an increased likelihood of DKA hospitalization in our multivariable analysis. The longer length of stay for Black patients despite similar presenting severity also exemplifies this complexity. For example, longer stays could result from explicit or implicit biases that lead providers to keep patients longer due to preconceived beliefs that the caregiver does not understand diabetes management. ${ }^{38}$ However, it also could be that the caregiver takes longer to complete diabetes education due to systemic barriers, such as work-related obligations during business hours when diabetes educators are present, or transportation and childcare needs. ${ }^{39}$ These barriers are often tied to socioeconomic status, making it more difficult for those in poverty to complete required education. We cannot conclude from our study the reasons for the difference in length of stay, just that differences exist. Moving forward, we suggest that design of interventions aimed at achieving health equity consider the complex interplay between health, healthcare systems, racism, and neighborhood context.

There were limitations to our study. First, given that this was an observational study, unmeasured confounders like patient or parental education and personal income could affect the strength of associations seen in our models. Second, although the CCHMC's Diabetes Center treats nearly all children with T1D in Hamilton County, some patients, or hospitalizations at other centers, could have been missed. Third, the demographics of Hamilton County may not generalize to other diabetes centers. Fourth, although more granular data-such as census block groups or blocks-are available from the US Census Bureau, we chose census tracts as our unit of analysis to reduce sampling error that emerges from the use of such smaller geographies. Last, we measured census tract poverty rather than other contextual markers of one's neighborhood, like vacant housing or the proportion of those receiving public benefits. However, these measures are often highly correlated with census tract poverty. ${ }^{40}$

\section{CONCLUSION}

Census tract poverty, race, and insurance were each associated with an increased likelihood of hospitalization for DKA. However, in a multivariable model, the effect of race was reduced so it was no longer significant, indicating that racial differences in DKA hospitalization are at least partially explained by differences in socioeconomic factors. The severity of DKA presentation did not differ by census tract poverty or insurance, although Black patients experienced differences in care despite similar markers of DKA severity. Addressing contextual factors - such as social determinants of health and racism-is warranted for future interventions that aim to eliminate equity gaps for T1D patients.

Disclosures: Dr Beck reports receiving grants from the National Institutes of Health and Robert Wood Johnson Foundation, paid to his institution, and consulting fees/honoraria from New York University, Montefiore Hospital, Xavier University, Randall Children's Hospital, and the American Academy of Pediatrics, all outside the submitted work. The other authors have nothing to disclose. Funding: Supported by the Cincinnati Children's Research Foundation's Diversity and Health Disparity Research Award (31-45310-586000-32-143064).

\section{References}

1. Mayer-Davis EJ, Lawrence JM, Dabelea D, et al. Incidence trends of type 1 and type 2 diabetes among youths, 2002-2012. N Engl J Med. 2017;376(15):1419-1429. https://doi.org/10.1056/NEJMoa1610187

2. Writing Group for the DCCT/EDIC Research Group, Orchard TJ, Nathan DM, et al. Association between 7 years of intensive treatment of type 1 diabetes and long-term mortality. JAMA. 2015;313(1):45-53. https://doi.org/10.1001/ jama.2014.16107

3. Wood JR, Miller KM, Maahs DM, et al. Most youth with type 1 diabetes in the T1D Exchange Clinic Registry do not meet American Diabetes Association or International Society for Pediatric and Adolescent Diabetes clinical guidelines. Diabetes Care. 2013;36(7):2035-2037. https://doi.org/10.2337/ dc12-1959

4. Benoit SR. Trends in diabetic ketoacidosis hospitalizations and in-hospital mortality-United States, 2000-2014. MMWR Morb Mortal Wkly Rep. 2018;67(12):362-365. https://doi.org/10.15585/mmwr.mm6712a3

5. Tieder JS, McLeod L, Keren R, et al. Variation in resource use and readmission for diabetic ketoacidosis in children's hospitals. Pediatrics. 2013;132(2):229236. https://doi.org/10.1542/peds.2013-0359

6. Moffett MA, Buckingham JC, Baker CR, Hawthorne G, Leech NJ. Patients' experience of admission to hospital with diabetic ketoacidosis and its psychological impact: an exploratory qualitative study. Practical Diabetes. 2013;30(5):203-207. https://doi.org/10.1002/pdi.1777

7. Maahs DM, Hermann JM, Holman N, et al. Rates of diabetic ketoacidosis: international comparison with 49,859 pediatric patients with type 1 diabetes from England, Wales, the U.S., Austria, and Germany. Diabetes Care. 2015;38(10):1876-1882. https://doi.org/10.2337/dc15-0780

8. Malik FS, Hall M, Mangione-Smith R, et al. Patient characteristics associated with differences in admission frequency for diabetic ketoacidosis in United States children's hospitals. J Pediatr. 2016;171:104-110. https://doi. 
org/10.1016/j.jpeds.2015.12.015

9. Manickam RN, Mu Y, Kshirsagar AV, Bang H. Area-level poverty and excess hospital readmission ratios. Am J Med. 2017;130(4):e153-e155. https:// doi.org/10.1016/j.amjmed.2016.08.047

10. Beck AF, Moncrief $T$, Huang $B$, et al. Inequalities in neighborhood child asthma admission rates and underlying community characteristics in one US county. J Pediatr. 2013;163(2):574-580.e1. https://doi.org/10.1016/ j.jpeds.2013.01.064

11. Everett E, Mathioudakis N. Association of area deprivation and diabetic ketoacidosis readmissions: comparative risk analysis of adults vs children with type 1 diabetes. J Clin Endocrinol Metab. 2019;104(8):3473-3480. https:// doi.org/10.1210/jc.2018-02232

12. U.S. Census Bureau. QuickFacts: Hamilton County, Ohio. Accessed March 2 2020. https://www.census.gov/quickfacts/hamiltoncountyohio

13. Witsch M, Kosteria I, Kordonouri O, et al. Possibilities and challenges of large international benchmarking in pediatric diabetology-the SWEET experience. Pediatr Diabetes. 2016;17(S23):7-15. https://doi.org/10.1111/ pedi.12432

14. U.S. Census Bureau. Glossary. Accessed April 22, 2021. https://www.census gov/programs-surveys/geography/about/glossary.html

15. U.S. Census Bureau. Geographic areas reference manual. Chapter 10: Cen sus tracts and block numbering areas. Accessed April 22, 2021. https://www. census.gov/programs-surveys/geography/guidance/geographic-areas-reference-manual.html

16. Krieger N, Chen JT, Waterman PD, Rehkopf DH, Subramanian SV. Painting a truer picture of US socioeconomic and racial/ethnic health inequalities: The Public Health Disparities Geocoding Project. Am J Public Health 2005:95(2):312-323. https://doi.org/10.2105/AJPH.2003.032482

17. Kitabchi AE, Umpierrez GE, Murphy MB, et al. Management of hypergly cemic crises in patients with diabetes. Diabetes Care. 2001;24(1):131-153. https://doi.org/10.2337/diacare.24.1.131

18. Lawson S, Redel JM, Smego A, et al. Assessment of a day hospital management program for children with type 1 diabetes. JAMA Netw Open 2020;3(3):e200347. https://doi.org/10.1001/jamanetworkopen.2020.0347

19. Andrist E, Riley CL, Brokamp C, Taylor S, Beck AF. Neighborhood poverty and pediatric intensive care use. Pediatrics. 2019;144(6):e20190748. https:// doi.org/10.1542/peds.2019-0748

20. Govan L, Maietti E, Torsney $\mathrm{B}$, et al. The effect of deprivation and $\mathrm{HbA} 1 \mathrm{c}$ on admission to hospital for diabetic ketoacidosis in type 1 diabetes. Diabetologia. 2012;55(9):2356-2360. https://doi.org/10.1007/s00125-012-2601-6

21. U.S. Department of Health and Human Services. Healthy People 2030 Social Determinants of Health. Accessed May 13, 2021. https://health.gov/ healthypeople/objectives-and-data/social-determinants-health

22. Berkowitz SA, Karter AJ, Corbie-Smith G, et al. Food insecurity, food "deserts," and glycemic control in patients with diabetes: a longitudinal analysis Diabetes Care. 2018:41(6):1188-1195. https://doi.org/10.2337/dc17-1981

23. Nguyen TM, Mason KJ, Sanders CG, Yazdani P, Heptulla RA. Targeting blood glucose management in school improves glycemic control in children with poorly controlled type 1 diabetes mellitus. J Pediatr. 2008;153(4):575-578. https://doi.org/10.1016/j.jpeds.2008.04.066

24. Izquierdo R, Morin PC, Bratt K, et al. School-centered telemedicine for children with type 1 diabetes mellitus. J Pediatr. 2009;155(3):374-379. https:// doi.org/10.1016/j.jpeds.2009.03.014

25. Council on Community Pediatrics and Committee on Native American Child Health. Policy statement-health equity and children's rights. Pediatrics. 2010;125(4):838-849. https://doi.org/10.1542/peds.2010-0235

26. Braveman P. What are health disparities and health equity? We need to be clear. Public Health Rep. 2014;129(suppl 2):5-8.

27. Palmas W, March D, Darakjy S, et al. Community health worker interventions to improve glycemic control in people with diabetes: a systematic review and meta-analysis. J Gen Intern Med. 2015;30(7):1004-1012. https:// doi.org/10.1007/s11606-015-3247-0

28. Lipman TH, Smith JA, Hawkes CP. Community health workers and the care of children with type 1 diabetes. J Pediatr Nurs. 2019;49:111-112. https:// doi.org/10.1016/j.pedn.2019.08.014

29. Bergenstal RM, Gal RL, Connor CG, et al. Racial differences in the relationship of glucose concentrations and hemoglobin A1c levels. Ann Intern Med. 2017;167(2):95-102. https://doi.org/10.7326/M16-2596

30. Agarwal S, Kanapka LG, Raymond JK, et al. Racial-ethnic inequity in young adults with type 1 diabetes. J Clin Endocrinol Metab. 2020;105(8):e2960-e2969. https://doi.org/10.1210/clinem/dgaa236

31. Addala A, Auzanneau M, Miller K, et al. A decade of disparities in diabe tes technology use and $\mathrm{HbA} 1 \mathrm{c}$ in pediatric type 1 diabetes: a transatlantic comparison. Diabetes Care. 2021;44(1):133-140. https://doi.org/10.2337/ dc20-0257

32. Lipman TH, Smith JA, Patil O, Willi SM, Hawkes CP. Racial disparities in treatment and outcomes of children with type 1 diabetes. Pediatr Diabetes. 2021:22(2):241-248. https://doi.org/10.1111/pedi.13139

33. Boyd RW, Lindo EG, Weeks LD, McLemore MR. On racism: a new standard for publishing on racial health inequities. Health Affairs. Accessed July 9 , 2020. https://www.healthaffairs.org/do/10.1377/hblog20200630.939347/full/

34. Jones CP. Levels of racism: a theoretic framework and a gardener's tale. Am J Public Health. 2000:90(8):1212-1215. https://doi.org/10.2105/AJPH.90.8.1212

35. Jacoby SF, Dong B, Beard JH, Wiebe DJ, Morrison CN. The enduring impact of historical and structural racism on urban violence in Philadelphia. Soc Sci Med. 2018;199:87-95. https://doi.org/10.1016/j.socscimed.2017.05.038

36. Bailey ZD, Krieger N, Agénor M, Graves J, Linos N, Bassett MT. Structural racism and health inequities in the USA: evidence and interventions. Lancet 2017;389(10077):1453-1463. https://doi.org/10.1016/S0140-6736(17)30569-X

37. Reardon SF, Fox L, Townsend J. Neighborhood income composition by household race and income, 1990-2009. Ann Am Acad Pol Soc Sci. 2015;660(1):78-97. https://doi.org/10.1177/0002716215576104

38. Cooper LA, Roter DL, Carson KA, et al. The associations of clinicians' im plicit attitudes about race with medical visit communication and patient ratings of interpersonal care. Am J Public Health. 2012;102(5):979-987. https:// doi.org/10.2105/AJPH.2011.300558

39. Lawton J, Waugh N, Noyes K, et al. Improving communication and recall of information in paediatric diabetes consultations: a qualitative study of parents' experiences and views. BMC Pediatr. 2015;15:67. https:// doi.org/10.1186/s12887-015-0388-6

40. Brokamp C, Beck AF, Goyal NK, Ryan P, Greenberg JM, Hall ES. Material community deprivation and hospital utilization during the first year of life: an urban population-based cohort study. Ann Epidemiol. 2019;30:37-43. https://doi.org/10.1016/j.annepidem.2018.11.008

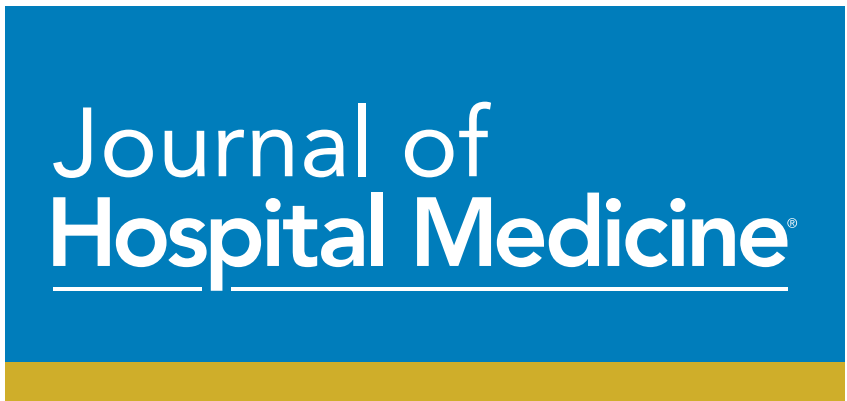

\section{journalofhospitalmedicine.com}

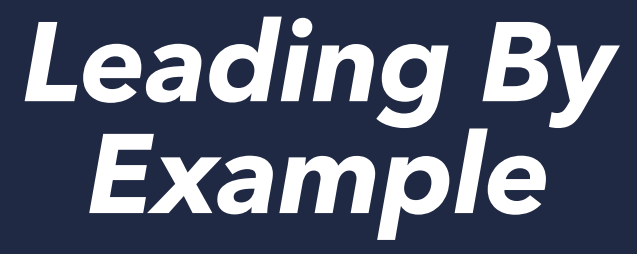

\#HowWeHospitalist 